\title{
Anthropometric risk factors for ovarian cancer in the NIH-AARP Diet and Health Study
}

\author{
Sebastian E. Baumeister ${ }^{1,2,3}$ (D) Inga Schlecht ${ }^{4} \cdot$ Britton Trabert $^{5} \cdot$ Michael Nolde ${ }^{1,2} \cdot$ Christa Meisinger $^{1,2}$. \\ Michael F. Leitzmann ${ }^{4}$
}

Received: 1 September 2020 / Accepted: 25 November 2020 / Published online: 22 January 2021

(c) The Author(s) 2021

\begin{abstract}
Objective Identifying potentially modifiable risk factors for ovarian cancer is essential for prevention because this cancer is predominantly detected at a late stage. Here, we estimated the relations of general adiposity and measures reflecting body fat distribution to the risk of epithelial ovarian cancer.

Methods We ascertained 683 ovarian epithelial cancers (343 high-grade serous, 141 non-high grade serous) among 145,575 women, aged 50-72 years (median follow-up 12.6 years), from the National Institutes of Health-American Association of Retired Persons (NIH-AARP) Diet and Health Study. Using Cox models, we estimated confounder-adjusted hazard ratios (HRs) and 95\% confidence intervals (CI) for associations of overall ovarian cancer, high-grade serous and non-high-grade serous carcinoma with body mass index, waist circumference, hip circumference, waist-hip ratio, waist-height ratio, body adiposity index, body shape index, and abdominal volume index.

Results Anthropometric measures were unrelated to overall ovarian cancer, high-grade serous cancer, and non-high-grade serous cancer. For example, the HR for overall ovarian cancer per standard deviation increment of body mass index at baseline was 0.98 (95\% CI 0.88-1.10). Similar associations were observed with measurements of body fat distribution.

Conclusion These results do not indicate that adult adiposity is associated with ovarian cancer risk in post-menopausal women.
\end{abstract}

Keywords Adiposity $\cdot$ Body fat $\cdot$ Obesity $\cdot$ Ovarian cancer risk

Sebastian E. Baumeister, Inga Schlecht, Christa Meisinger and Michael F. Leitzmann contributed equally to this work.

Supplementary Information The online version contains supplementary material available at https://doi.org/10.1007/s1055 2-020-01377-y.

Sebastian E. Baumeister

s.baumeister@unika-t.de

1 Chair of Epidemiology, Ludwig-Maximilians-Universität München, UNIKA-T Augsburg, Neusässer Str. 47, 86156 Augsburg, Germany

2 Independent Research Group Clinical Epidemiology, Helmholtz Zentrum München, German Research Center for Environmental Health, Neuherberg, Germany

3 University of Münster, Münster, Germany

4 Department of Epidemiology and Preventive Medicine, University of Regensburg, Regensburg, Germany

5 Division of Cancer Epidemiology and Genetics, National Cancer Institute, National Institutes of Health, Bethesda, MD, USA

\section{Introduction}

Ovarian cancer is the fifth most common cause of cancer death in women in North America and western Europe [1]. Meta-analyses and pooled observational studies suggest that obesity may be positively related to ovarian cancer risk [2-5]. The World Cancer Research Fund/American Institute for Cancer Research concluded that the evidence for the link between obesity and increased ovarian cancer risk is probable [6], and an umbrella review graded the evidence as suggestive [3]. A growing body of research further suggests that body composition plays an important role in site-specific cancer development [7-9]. Previous studies indicate that anthropometric measures of general obesity and body fat distribution may be differentially related to ovarian cancer risk; however, results have been conflicting [4, 5, 10-12]. We conducted a cohort study among post-menopausal women using data from the National Institutes of Health-American Association of Retired Persons (NIH-AARP) Diet and 
Health Study to provide further insights into the association between body fatness and subtype-specific epithelial ovarian cancer risk by comparing indicators of general obesity and body fat distribution.

\section{Methods}

\section{Study population}

The NIH-AARP Diet and Health Study is a prospective cohort study of persons in the U.S. [13]. At baseline (1995-1996), 3.5 million AARP members aged 50-71 years who resided in six states (California, Florida, Louisiana, New Jersey, North Carolina, and Pennsylvania) and two metropolitan areas (Atlanta, Georgia; and Detroit, Michigan) were invited to complete a questionnaire on

$\left(2 \mathrm{~cm} \times\right.$ waist circumference $\left.{ }^{2}(\mathrm{~cm})+0.7 \mathrm{~cm} \times(\text { waist circumference }(\mathrm{cm})-\text { hip circumference }(\mathrm{cm}))^{2}\right) / 1,000$.

\section{Assessment of anthropometric measures}

Self-reported height and weight were obtained from the baseline questionnaire. Body mass index was calculated as weight $(\mathrm{kg})$ divided by the square of height (in meters). In the second questionnaire, participants were instructed to measure their waist circumference and hip circumference using a tape measure to the nearest 0.25 inch while standing. Waist circumference was to be measured 1 inch above the navel if this was not the waistline. Hip circumference was defined as the largest circumference between the upper edge of the pelvis and the femur. Waist-hip ratio was calculated by dividing waist circumference $(\mathrm{cm})$ by hip circumference $(\mathrm{cm})$, and waist-height ratio was calculated as waist circumference $(\mathrm{cm}) /$ height $(\mathrm{cm})$. The body adiposity index was calculated as hip circumference $(\mathrm{cm}) /$ height $^{1.5}(\mathrm{~m})-18$ [14]. The body shape index was based on waist circumference $(\mathrm{cm}) /$ body mass index ${ }^{\frac{2}{3}} \times$ height $^{2}(\mathrm{~m})$

[15]. The abdominal volume index was also quantified [16]: demographic, diet, and lifestyle characteristics. Questionnaires were completed satisfactorily by 566,398 participants. Six months after completing the baseline questionnaire, a second questionnaire was mailed to living participants who did not have a self-reported colon, breast, or prostate cancer at baseline to collect additional information. Self-reported weight and height were collected on the baseline questionnaire. Self-reported waist circumference and hip circumference were assessed on the second questionnaire. We excluded male participants $(n=339,666)$; those with unknown cancer or previous diagnosis of cancer other than non-melanoma skin cancer at baseline before completion of the second questionnaire $(n=16,300)$; those with bilateral oophorectomy and unknown oophorectomy status $(n=57,047)$; those with no information on height, weight, waist circumference, or hip circumference $(n=95,764)$; and subjects with body mass index less than $18.5 \mathrm{~kg} / \mathrm{m}^{2}$ or more than $65 \mathrm{~kg} / \mathrm{m}^{2}(n=3,528)$. Our final analytical datasets included 145,575 women for the analysis on body mass index, 60,999 for waist circumference, 60,826 for hip circumference, 60,597 for waist-to-hip ratio, 60,999 for waist-to-height ratio, 60,826 for body adiposity index, 60,999 for body shape index, and 60,597 for abdominal adiposity index. The Special Studies Institutional Review Board of the U.S. National Cancer Institute approved the study [13]. All participants gave informed consent by virtue of completing and returning the baseline questionnaire.

\section{Definition of cancer outcomes}

Diagnoses of ovarian cancer were ascertained through 31 September 2011, via linkage to state cancer registries of the eight recruitment areas where the study participants were most likely to relocate: Arizona, Nevada, and Texas. This approach has been estimated to yield a sensitivity of $90 \%$ and a specificity of nearly $100 \%$ [17]. Newly diagnosed ovarian cancer cases were identified using the International Classification of Diseases for Oncology (ICDO-3), topography (C56.9), and morphology codes (8441, $8460,8461,8470,8471,8480,8481,8380,8381,8560$, $8570,8310,8313,8010,8020,8021,8050,8070,8120$, $8140,8255,8260,8323,8440,8450,8562,9000)$ [18]. The high-grade serous group included all invasive serous cancers except low grade $[19,20]$. The non-high-grade group included all serous cancers and invasive mucinous, endometrial, and clear cell cancers [20]. Borderline tumors were excluded from this study.

\section{Baseline confounders}

We controlled for several baseline participant characteristics that were assumed to cause adiposity or ovarian cancer $[3,5,21-23]$. We assumed that direct causes of the exposure or outcome, excluding possible instrumental variables, would identify a sufficient set of confounders [24]. Potential confounding variables included age, education (no school 
degree/unknown, primary school, technical school/secondary, university), participants' self-reported information on race/ethnicity (none-Hispanic white, other), cigarette smoking (never, current $<15$ cigarettes per day, current $\geq 15$ cigarettes/day, former $<10$ years, former $\geq 10$ years), alcohol consumption (in grams of pure alcohol per day), parity ( 0 , $1, \geq 2$ children), age at menarche ( $\leq 12$ years, $>12$ years), family history of ovarian cancer, oral contraceptive use, and menopausal hormone therapy.

\section{Statistical analysis}

Cox proportional hazards regression was used for estimating adjusted hazard ratios (HRs) and 95\% confidence intervals (CIs) for overall ovarian cancer and ovarian cancer subtypes. Time of study entry was age at baseline (or second questionnaire) and exit time was age at cancer diagnosis or the last date at which follow-up was considered complete. Athropometric measures were modeled as a continuous and categorical metric. After confirming that the linearity assumption was met by testing cubic spline transformations [25], HRs were estimated per standard deviation (1-SD) increase in anthropometric measures. Body mass index $\left(\mathrm{kg} / \mathrm{m}^{2}\right)$ was further grouped as normal weight $(18.5$ to $<25)$, overweight $(25$ to $<30)$, and obese $(\geq 30)$. Waist circumference and other anthropometric measures were categorized according to quartiles. Models were stratified by 5 -year age groups to minimize departure from proportionality and adjusted for education, race, smoking, alcohol consumption (modeled continuously using restricted cubic splines [25]), parity, age at menarche, family history of ovarian cancer, oral contraceptive use, and menopausal hormone therapy.

The improvement in predictive accuracy after adding anthropometric measures to a null model (including race, age, education, smoking, alcohol consumption, parity, family history of ovarian cancer, oral contraceptive use and menopausal hormone therapy) was evaluated in terms of explained variation $\left(R^{2}\right)$ [26], the Bayes Information Criterion (BIC), and model discrimination using Harrell's C-index [25] derived from flexible parametric models [27]. $p$ values for the difference between Harrell's $C$ indices of models with and without anthropometric indicators were computed using the method proposed by Antolini et al. [28]. We used 1,000 bootstrap replications to perform internal validations and to correct $R^{2}$, BIC, and Harrell's C-indices for optimism [25].

In a sensitivity analysis, we used regression calibration for self-reported body mass index, waist circumference, and hip circumference to assess possible regression dilution bias [29]. Because replicate measurements were not available, we applied published reliability coefficients [30-33], ranging from 0.5 to 0.9 . A further threat to the validity of our estimates is potential unobserved confounding by undiagnosed ovarian cancer (often referred to as "reverse causation" [34]) if these conditions are symptomatic enough to induce a change in body weight. We, therefore, assumed 3 years of minimum latent period required for weight change due to unobserved disease to affect the outcome and excluded events that occurred during this time [35]. The statistical analysis was performed using Stata 15.1.

\section{Results}

In the analytical sample of 145,575 women, the mean (SD) age at baseline was 61.8 (5.4) years. During a median followup time of 12.6 years, participants contributed 1,897,323 person-years and 683 ovarian cancer (343 high-grade serous) cases occurred. The baseline characteristics of the analytical sample are provided in Table 1.

Neither body mass index nor other anthropometric measurements were associated with the risk of ovarian cancer (Table 2). For example, HRs for overall ovarian cancer per 1-SD increment in body mass index, waist circumference, hip circumference, waist-hip ratio, and waist-height ratio were 0.98 (95\% CI 0.88-1.10), 1.08 (95\% CI 0.91-1.27), 1.06 (95\% CI $0.90-1.25), 1.03$ (95\% CI 0.89-1.21), and 1.08 (95\% CI 0.92-1.27), respectively. In categorical analyses, the HRs for comparing obese and normal weight body mass index groups, and the highest and lowest quartiles of waist circumference, hip circumference, waist-hip ratio, and waist-height ratio were 1.11 (95\% CI 0.91-1.36), 1.17 (95\% CI 0.84-1.62), 1.28 (95\% CI 0.94-1.75), 1.35 (95\% CI 0.97; 1.89 ), and 1.18 (95\% CI 0.85-1.64), respectively. No associations were observed for high-grade serous carcinomas. The accuracy of models predicting ovarian cancer risk was not improved after adding anthropometric measures (Table 3).

Sensitivity analysis indicated that HRs could have been attenuated towards the null because of measurement error in self-reported anthropometric measurements. For example, the unadjusted HR for per SD of waist circumference and overall ovarian cancer was 1.08 (95\% CI 0.91-1.27), but after accounting for potential regression dilution bias, assuming an attenuation factor of 0.7, the HR was 1.11 (95\% CI 0.91-1.30) (Supplementary Table 1). The associations were virtually unchanged when events occurring during the first three years of follow-up were excluded (Supplementary Table 2).

\section{Discussion}

This study examined the association of indicators of general obesity and body fat distribution with ovarian cancer risk using a large U.S. prospective cohort study of 
Table 1 Baseline characteristics of ovarian cancer cases among 145,575 women in the NIH-AARP Study

All study subjects No ovarian cancer Ovarian cancer Histological subtypes

High-grade serous Non-high-grade serous

\begin{tabular}{|c|c|c|c|c|c|}
\hline Age (years) & $61.8(5.4)$ & $61.8(5.4)$ & $62.7(5.4)$ & $62.4(5.4)$ & $61.9(5.7)$ \\
\hline \multicolumn{6}{|l|}{ Education $(n(\%))$} \\
\hline$<12$ years & $8,259(5.6)$ & $8,223(5.6)$ & $36(5.1)$ & $11(3.1)$ & $7(4.8)$ \\
\hline 12 years & $37,549(25.6)$ & $37,374(26.6)$ & $175(24.9)$ & $85(24.2)$ & $43(29.4)$ \\
\hline$>12$ years & $100,736(68.7)$ & $100,243(68.7)$ & $493(70.0)$ & 255 (72.7) & $96(65.8)$ \\
\hline \multicolumn{6}{|l|}{$\operatorname{Race}(n(\%))$} \\
\hline Non-hispanic white & $137,456(91.2)$ & $136,763(91.3)$ & $693(94.93)$ & $355(96.2)$ & $141(94.6)$ \\
\hline Non-hispanic black & $7,883(7.9)$ & $7,864(5.3)$ & $19(2.6)$ & $4(1.1)$ & $6(4.0)$ \\
\hline Hispanic/other & $5,199(5.2)$ & $5,181(3.5)$ & $18(2.5)$ & $10(2.7)$ & $2(1.3)$ \\
\hline Body mass index $\left(\mathrm{kg} / \mathrm{m}^{2}\right)$ & $26.8(5.5)$ & $26.8(5.5)$ & $26.7(5.5)$ & $26.2(4.8)$ & $26.4(5.2)$ \\
\hline 18.5 to $<25(n(\%))$ & $67,266(44.7)$ & $66,936(44.7)$ & $330(45.2)$ & $179(48.5)$ & $64(43.0)$ \\
\hline 25 to $<30(n(\%))$ & 49,207 (32.7) & $48,979(32.2)$ & $228(31.2)$ & $115(31.2)$ & $53(35.6)$ \\
\hline $30+(n(\%))$ & $34,065(22.6)$ & $33,893(22.6)$ & $172(23.6)$ & $75(20.3)$ & $32(21.5)$ \\
\hline Waist circumference $(\mathrm{cm})$ & $84.6(13.2)$ & $84.6(13.2)$ & $85.3(13.2)$ & $84.0(11.6)$ & $86.6(15.8)$ \\
\hline Quartile $1(n(\%))$ & $15,346(25.0)$ & $15,268(24.4)$ & $78(25.2)$ & $40(25.1)$ & $16(28.8)$ \\
\hline Quartile $2(n(\%))$ & $16,600(25.0)$ & $16,533(26.5)$ & 67 (21.6) & $41(25.8)$ & $5(8.9)$ \\
\hline Quartile $3(n(\%))$ & $15,618(25.0)$ & $15,538(24.9))$ & $80(25.8)$ & $41(25.8)$ & $17(30.4)$ \\
\hline Quartile $4(n(\%))$ & $15,247(25.0)$ & $15,162(24.3)$ & $85(27.4)$ & $37(23.3)$ & $18(32.1)$ \\
\hline Hip circumference $(\mathrm{cm})$ & $103.9(11.5)$ & $103.9(11.5)$ & $104.5(11.4)$ & $103.4(10.2)$ & $104.3(11.9)$ \\
\hline Quartile $1(n(\%))$ & $19,006(25.0)$ & $18,919(30.4)$ & $87(28.1)$ & $49(31.0)$ & $14(25.0)$ \\
\hline Quartile $2(n(\%))$ & $13,197(25.0)$ & $13,128(21.1)$ & $69(22.3)$ & $37(23.4)$ & $11(19.6)$ \\
\hline Quartile $3(n(\%))$ & $15,192(25.0)$ & $15,126(24.3$ & $66(21.3)$ & $33(20.9)$ & $12(21.4)$ \\
\hline Quartile $4(n(\%))$ & $15,231(25.0)$ & $15,143(24.3)$ & $88(28.4)$ & $29(24.7)$ & $19(33.9)$ \\
\hline Waist-hip ratio & $0.81(0.08)$ & $0.81(0.08)$ & $0.81(0.08)$ & $0.81(0.07)$ & $0.81(0.09)$ \\
\hline Quartile $1(n(\%))$ & $15,525(25.0)$ & $15,454(24.9)$ & $71(22.9)$ & $35(22.2)$ & $17(30.4)$ \\
\hline Quartile $2(n(\%))$ & $15,704(25.0)$ & $15,628(25.2)$ & $76(24.6)$ & $44(27.9)$ & $7(12.5)$ \\
\hline Quartile $3(n(\%))$ & $15,833(25.0)$ & $15,755(25.4)$ & $78(25.2)$ & $40(25.3)$ & $13(23.2)$ \\
\hline Quartile $4(n(\%))$ & $15,317(25.0)$ & $15,233(24.5)$ & $84(27.2)$ & $39(24.7)$ & $19(33.9)$ \\
\hline Waist-height ratio & $0.52(0.08)$ & $0.51(0.08)$ & $0.52(0.0 .8)$ & $0.51(0.07)$ & $0.52(0.10)$ \\
\hline Quartile $1(n(\%))$ & $15,360(25.0)$ & $15,283(24.5)$ & $77(24.8)$ & $36(22.6)$ & $17(30.4)$ \\
\hline Quartile $2(n(\%))$ & $16,183(25.0)$ & $16,116(25.8)$ & 67 (21.6) & $40(25.2)$ & $7(12.5)$ \\
\hline Quartile $3(n(\%))$ & $15,811(25.0)$ & $15,731(25.2)$ & $80(25.8)$ & $45(28.3)$ & $14(25.0)$ \\
\hline Quartile $4(n(\%))$ & $15,457(25.0)$ & $15,371(24.6)$ & $86(27.7)$ & $38(23.9)$ & $18(32.1)$ \\
\hline Body adiposity index & $31.9(5.8)$ & $31.8(5.8)$ & $32.2(6.0)$ & $31.7(5.3)$ & $32.1(5.9)$ \\
\hline Quartile $1(n(\%))$ & $15,045(25.0)$ & $14,976(24.0)$ & $69(22.3)$ & $34(21.5)$ & $11(19.6)$ \\
\hline Quartile $2(n(\%))$ & $16,882(25.0)$ & $16,800(27.0))$ & $82(26.5)$ & $51(32.3)$ & $11(19.6)$ \\
\hline Quartile $3(n(\%))$ & $15,524(25.0)$ & $15,445(24.8)$ & $79(25.5)$ & $40(25.3)$ & $20(35.7)$ \\
\hline Quartile $4(n(\%))$ & $15,175(25.0)$ & $15,095(24.2)$ & $80(25.8)$ & $33(20.9)$ & $14(25.0)$ \\
\hline Body shape index & $0.01(0.003)$ & $0.01(0.003)$ & $0.01(0.003)$ & $0.01(0.003)$ & $0.01(0.003)$ \\
\hline Quartile $1(n(\%))$ & $15,302(25.0)$ & $15,226(24.4)$ & $76(24.5)$ & $33(20.8)$ & $13(23.2)$ \\
\hline Quartile $2(n(\%))$ & $15,958(25.0)$ & $15,884(25.4)$ & $74(23.9)$ & $36(22.6)$ & $17(20.4)$ \\
\hline Quartile $3(n(\%))$ & $16,294(25.0)$ & $16,212(25.9)$ & $82(26.5)$ & $46(28.9)$ & $18(32.1)$ \\
\hline Quartile $4(n(\%))$ & $15,257(25.0)$ & $15,179(24.3)$ & $78(25.2)$ & $44(27.7)$ & $8(14.3)$ \\
\hline Abdominal volume index & $15.0(4.7)$ & $15.0(4.7)$ & $15.2(4.6)$ & $14.7(3.9)$ & $15.5(5.4)$ \\
\hline Quartile $1(n(\%))$ & $15,317(25.0)$ & $15,237(24.6)$ & $80(25.9)$ & $41(26.0)$ & $16(28.6)$ \\
\hline Quartile $2(n(\%))$ & $16,005(25.0)$ & $15,942(25.7)$ & $63(20.4)$ & $39(24.7)$ & $5(8.9)$ \\
\hline Quartile $3(n(\%))$ & $15,711(25.0)$ & $15,632(25.2)$ & $79(25.6)$ & $39(24.7)$ & $17(30.4)$ \\
\hline Quartile $4(n(\%))$ & $15,346(25.0)$ & $15,259(25.6)$ & $87(28.2)$ & $39(24.7)$ & $18(32.1)$ \\
\hline
\end{tabular}


Table 1 (continued)

All study subjects No ovarian cancer Ovarian cancer Histological subtypes

$\overline{\text { High-grade serous Non-high-grade serous }}$

\begin{tabular}{|c|c|c|c|c|c|}
\hline \multicolumn{6}{|l|}{ Smoking status $(n(\%))$} \\
\hline Never smoked & $68,479(45.5)$ & $68,118(45.5)$ & $361(49.5)$ & $202(54.7)$ & $64(42.9)$ \\
\hline Former smoker, $\leq 20$ cigarettes per day & $41,527(27.6)$ & $41,323(27.6)$ & 204 (27.9) & $87(23.6)$ & $56(37.6)$ \\
\hline Former smoker, $>20$ cigarettes per day & $18,884(12.5$ & $18,803(12.6)$ & $81(11.1)$ & $38(10.5)$ & $14(9.4)$ \\
\hline Current smoker, $\leq 20$ cigarettes per day & $15,774(10.5$ & $15,709(10.5)$ & $65(8.8)$ & $33(8.9)$ & $14(9.4)$ \\
\hline Current smoker, $>20$ cigarettes per day & $5,874(3.9)$ & $5,855(3,9)$ & $19(2.6)$ & $9(2.4)$ & $1(0.7)$ \\
\hline Alcohol consumption (g/day) & $6.2(17.3)$ & $6.2(17.3)$ & $6.0(14.7)$ & $5.4(9.8)$ & $5.5(13.4)$ \\
\hline \multicolumn{6}{|l|}{ Parity $(n(\%))$} \\
\hline Never had a child & $22,954(15.3)$ & $22,819(5.3)$ & $135(18.5)$ & $63(17.1)$ & $39(26.4)$ \\
\hline 1 child & $15,342(10.2)$ & $15,255(10.3)$ & $87(11.9)$ & $44(11.9)$ & $18(12.2)$ \\
\hline 2 and more children & $111,501(74.4)$ & $110,994(74.4)$ & $507(69.4)$ & $262(70.0)$ & $91(51.5)$ \\
\hline \multicolumn{6}{|l|}{ Age at menarche $(n(\%))$} \\
\hline$\leq 12$ years & $72,734(48.5)$ & $72,375(48.5)$ & $359(49.4)$ & $191(51.8)$ & $67(45.6)$ \\
\hline$>12$ years & $77,150(51.5)$ & $76,782(51.5)$ & $368(50.6)$ & $178(48.2)$ & $80(54.4)$ \\
\hline Family history of ovarian cancer $(n(\%))$ & $9,154(6.1)$ & $9,101(6.1)$ & $53(7.3)$ & $22(5.9)$ & $13(8.7))$ \\
\hline Ever oral contraceptive use $(n(\%))$ & $60,739(40.7)$ & $60,500(40.6)$ & $239(33.1)$ & $115(31.3)$ & $64(43.2)$ \\
\hline $\begin{array}{l}\text { Ever hormone replacement therapy }(n \\
(\%))\end{array}$ & $69,739(46.3)$ & $69,370(46.3)$ & $369(50.6)$ & $201(54.5)$ & $70(47.0)$ \\
\hline
\end{tabular}

NIH-AARP, NIH-AARP Diet and Health Study. Entries are means (standard deviation) for continuous variables and percent values for categorical variables

post-menopausal women. We found no association of anthropometric measures with the risk of overall ovarian cancer, high-grade serous, or non-high-grade ovarian cancers. Overall, our analysis does not support the hypothesis that central adiposity or measures of body fat distribution improve the prediction of ovarian cancer risk in post-menopausal women.

A larger body of research examined the association between obesity and ovarian cancer risk. However, the findings of more than 30 epidemiologic studies have been weak and mixed [36]. Several meta-analyses and pooled analyses reported weak positive associations between adult body mass index and ovarian cancer risk, noting substantial between-study heterogeneity with weaker associations in prospective than case-control studies [2, 6, 37]. A systematic review from the World Cancer Research Fund and the American Institute for Cancer Research included 28 prospective studies on ovarian cancer and calculated a summary relative risk for a 5-unit increment in body mass index of 1.07 (95\% CI 1.03-1.11) [2, 6]. The effect size was similar in the post-menopausal group but it was less precise (relative risk per $5 \mathrm{~kg} \mathrm{~m}^{-2}=1.07$; 95\% CI 1.00-1.14). Results from the 2013 Ovarian Cancer Association Consortium [4] pooled analysis of case-control studies found that the positive association with body mass index was stronger in premenopausal women. The heterogeneity of findings reported here and previously could be explained by menopausal status and higher statistical efficacy of meta-analysis. Indeed, our HRs for body mass index and waist circumference overlap with the HR's CIs of the World Cancer Research Fund/ American Institute for Cancer Research meta-analysis [2, 6], which indicates that there might be chance variation of estimates. Few studies have examined how different measures of body fat distribution are related to ovarian cancer and its subtypes [10-12]. Existing cohort studies found no association of waist circumference and waist-hip ratio and ovarian cancer risk $[2,6,10]$. Similar to previous studies [4, 5], the present study found no notable differences between histotypes. In contrast, a Mendelian randomization study suggested that obesity might cause non-high-grade but not high-grade ovarian cancer [20].

The present study has several limitations. It relied on self-reported anthropometric data and potential measurement error could have attenuated the observed associations. Anthropometric measurements were taken when most study participants had reached menopause. However, pre-menopausal anthropometric risk factors might be more strongly related to ovarian cancer risk. A Mendelian randomization study suggested a positive association with higher body mass index with risk of ovarian cancer among pre-menopausal women, but not for post-menopausal women [38]. Unfortunately, we could not test whether the association between anthropometric markers and ovarian cancer risk was modified by menopause status. More studies on body 
Table 2 Association of general obesity and indicators of body fat distribution with ovarian cancer in NIH-AARP

\begin{tabular}{|c|c|c|c|}
\hline & Ovarian cancer risk & High-grade serous & Non-high-grade serous \\
\hline Body mass index $(n=145,575)$, number of cases & 683 & 343 & 141 \\
\hline HR per SD $(95 \% \mathrm{CI})$ & $0.98(0.88 ; 1.10)$ & $0.91(0.77 ; 1.08)$ & $0.92(0.71 ; 1.19)$ \\
\hline \multicolumn{4}{|l|}{ HR, categorical } \\
\hline 18.5 to $<25(n(\%))$ & Reference & Reference & Reference \\
\hline 25 to $<30(n(\%))$ & $0.97(0.81 ; 1.16)$ & $0.94(0.73 ; 1.20)$ & $1.13(0.77 ; 1.65)$ \\
\hline$\geq 30(n(\%))$ & $1.11(0.91 ; 1.36)$ & $0.91(0.68 ; 1.22)$ & $1.03(0.65 ; 1.63)$ \\
\hline Joint $p$ value & 0.418 & 0.772 & 0.814 \\
\hline Waist circumference $(n=60,999)$, number of cases & 295 & 151 & 54 \\
\hline HR per SD $(95 \% \mathrm{CI})$ & $1.08(0.91 ; 1.27)$ & $1.00(0.79 ; 1.27)$ & $1.10(0.76 ; 1.60)$ \\
\hline \multicolumn{4}{|l|}{$\mathrm{HR}$, categorical } \\
\hline Quartile 1 & Reference & Reference & Reference \\
\hline Quartile 2 & $0.82(0.59 ; 1.16)$ & $1.09(0.69 ; 1.70)$ & $0.30(0.11 ; 0.81)$ \\
\hline Quartile 3 & $1.06(0.76 ; 1.46)$ & $1.16(0.74 ; 1.84)$ & $0.97(0.48 ; 1.99)$ \\
\hline Quartile 4 & $1.17(0.84 ; 1.62)$ & $1.07(0.66 ; 1.73)$ & $1.22(0.61 ; 2.47)$ \\
\hline Joint $p$ value & 0.226 & 0.932 & 0.099 \\
\hline Hip circumference $(n=60,826)$, number of cases & 295 & 150 & 54 \\
\hline HR per SD $(95 \% \mathrm{CI})$ & $1.06(0.90 ; 1.25)$ & $0.98(0.77 ; 1.24)$ & $1.02(0.70 ; 1.49)$ \\
\hline \multicolumn{4}{|l|}{ HR, categorical } \\
\hline Quartile 1 & Reference & Reference & Reference \\
\hline Quartile 2 & $1.07(0.77 ; 1.48)$ & $1.07(0.69 ; 1.66)$ & $1.13(0.51 ; 2.50)$ \\
\hline Quartile 3 & $0.91(0.65 ; 1.27)$ & $0.83(0.52 ; 1.31)$ & $1.00(0.45 ; 2.20)$ \\
\hline Quartile 4 & $1.28(0.94 ; 1.75)$ & $1.06(0.68 ; 1.65)$ & $1.67(0.81 ; 3.43)$ \\
\hline Joint $p$ value & 0.203 & 0.712 & 0.448 \\
\hline Waist-hip ratio $(n=60,597)$, number of cases & 294 & 150 & 54 \\
\hline $\mathrm{HR}$ per $\mathrm{SD}(95 \% \mathrm{CI})$ & $1.03(0.89 ; 1.21)$ & $1.03(0.82 ; 1.29)$ & $1.02(0.70 ; 1.49)$ \\
\hline \multicolumn{4}{|l|}{$\mathrm{HR}$, categorical } \\
\hline Quartile 1 & Reference & Reference & Reference \\
\hline Quartile 2 & $1.16(0.83 ; 1.63)$ & $1.44(0.91 ; 2.28)$ & $0.38(0.15 ; 0.98)$ \\
\hline Quartile 3 & $1.15(0.82 ; 1.61)$ & $1.22(0.75 ; 1.97)$ & $0.84(0.40 ; 1.76)$ \\
\hline Quartile 4 & $1.35(0.97 ; 1.89)$ & $1.38(0.85 ; 2.24)$ & $1.31(0.66 ; 2.61)$ \\
\hline Joint $p$ value & 0.362 & 0.435 & 0.069 \\
\hline Waist-height ratio $(n=60,999)$, number of cases & 295 & 151 & 54 \\
\hline HR per SD $(95 \% \mathrm{CI})$ & $1.08(0.92 ; 1.27)$ & $1.00(0.79 ; 1.27)$ & $1.13(0.78 ; 1.64)$ \\
\hline \multicolumn{4}{|l|}{ HR, categorical } \\
\hline Quartile 1 & Reference & Reference & Reference \\
\hline Quartile 2 & $0.86(0.61 ; 1.20)$ & $1.22(0.76 ; 1.94$ & $0.35(0.14 ; 0.89)$ \\
\hline Quartile 3 & $1.06(0.76 ; 1.47)$ & $1.42(0.89 ; 2.25)$ & $0.80(0.38 ; 1.67)$ \\
\hline Quartile 4 & $1.18(0.85 ; 1.64)$ & $1.22(0.74 ; 2.00)$ & $1.16(0.58 ; 2.33)$ \\
\hline Joint $p$ value & 0.304 & 0.530 & 0.080 \\
\hline Body adiposity index $(n=60,826)$, number of cases & 294 & 150 & 54 \\
\hline HR per SD $(95 \% \mathrm{CI})$ & $1.07(0.91 ; 1.26)$ & $0.98(0.77 ; 1.25)$ & $1.06(0.76 ; 1.55)$ \\
\hline \multicolumn{4}{|l|}{ HR, categorical } \\
\hline Quartile 1 & Reference & Reference & Reference \\
\hline Quartile 2 & $1.00(0.72 ; 1.39)$ & $1.33(0.85 ; 2.08)$ & $0.92(0.40 ; 2.13)$ \\
\hline Quartile 3 & $1.06(0.76 ; 1.48)$ & $1.13(0.70 ; 1.82)$ & $1.78(0.84 ; 3.77)$ \\
\hline Quartile 4 & $1.14(0.81 ; 1.48)$ & $0.99(0.59 ; 1.65)$ & $1.27(0.55 ; 2.93)$ \\
\hline Joint $p$ value & 0.850 & 0.508 & 0.279 \\
\hline Body shape index $(n=60,999)$, number of cases & 294 & 151 & 54 \\
\hline HR per SD $(95 \% \mathrm{CI})$ & $0.98(0.83 ; 1.16)$ & $1.08(0.85 ; 1.36)$ & $0.82(0.55 ; 1.21)$ \\
\hline
\end{tabular}


Table 2 (continued)

\begin{tabular}{|c|c|c|c|}
\hline & Ovarian cancer risk & High-grade serous & Non-high-grade serous \\
\hline \multicolumn{4}{|l|}{ HR, categorical } \\
\hline Quartile 1 & Reference & Reference & Reference \\
\hline Quartile 2 & $0.84(0.60 ; 1.17)$ & $0.92(0.56 ; 1.50)$ & $1.16(0.55 ; 2.44)$ \\
\hline Quartile 3 & $0.94(0.68 ; 1.30)$ & $1.18(0.74 ; 1.88)$ & $1.17(0.56 ; 2.46)$ \\
\hline Quartile 4 & $0.93(0.67 ; 1.30)$ & $1.20(0.75 ; 1.93)$ & $0.56(0.23 ; 1.40)$ \\
\hline Joint $p$ value & 0.787 & 0.611 & 0.342 \\
\hline Abdominal volume index $(n=60,597)$, number of cases & 295 & 150 & \\
\hline HR per $\mathrm{SD}(95 \% \mathrm{CI})$ & $1.07(0.91 ; 1.25)$ & $0.98(0.77 ; 1.25)$ & $1.13(0.79 ; 1.61)$ \\
\hline \multicolumn{4}{|l|}{ HR, categorical } \\
\hline Quartile 1 & Reference & Reference & Reference \\
\hline Quartile 2 & $0.78(0.55 ; 1.10)$ & $1.04(0.66 ; 1.63)$ & $0.31(0.11 ; 0.84)$ \\
\hline Quartile 3 & $1.00(0.73 ; 1.39)$ & $1.06(0.67 ; 1.68)$ & $0.96(0.47 ; 1.97)$ \\
\hline Quartile 4 & $1.15(0.83 ; 1.59)$ & $1.09(0.68 ; 1.74)$ & $1.21(0.60 ; 2.44)$ \\
\hline Joint $p$ value & 0.155 & 0.988 & 0.061 \\
\hline
\end{tabular}

NIH-AARP, NIH-AARP Diet and Health Study. HR (hazard ratio) from age-group stratified multivariable Cox model adjusted for education, race, smoking, alcohol consumption, parity, age at menarche, family history of ovarian cancer, oral contraceptive use, and menopausal hormone therapy. Joint $p$ value from Wald test of all exposure dummy variables

Table 3 General obesity and indicators of body fat distribution for prediction of ovarian cancer

\begin{tabular}{|c|c|c|c|c|c|c|c|c|c|}
\hline & $\begin{array}{l}\text { Baseline } \\
\text { model }\end{array}$ & $\begin{array}{l}\text { Body mass } \\
\text { index }\end{array}$ & $\begin{array}{l}\text { Waist cir- } \\
\text { cumference }\end{array}$ & $\begin{array}{l}\text { Hip circum- } \\
\text { ference }\end{array}$ & $\begin{array}{l}\text { Waist-to-hip } \\
\text { ratio }\end{array}$ & $\begin{array}{l}\text { Waist-to- } \\
\text { height ratio }\end{array}$ & $\begin{array}{l}\text { Body adi- } \\
\text { posity index }\end{array}$ & $\begin{array}{l}\text { Body shape } \\
\text { index }\end{array}$ & $\begin{array}{l}\text { Abdominal } \\
\text { volume } \\
\text { index }\end{array}$ \\
\hline \multicolumn{10}{|c|}{ Ovarian cancer } \\
\hline $\begin{array}{l}\text { Adjusted } \\
R^{2}\end{array}$ & 0.059 & 0.059 & 0.071 & 0.068 & 0.066 & 0.072 & 0.068 & 0.069 & 0.068 \\
\hline $\mathrm{BIC}$ & $16,349.94$ & $16,361.70$ & $6,650.949$ & $6,629.188$ & $6,627.817$ & $6,650.704$ & $6,628.799$ & $6,652.352$ & $6,626.746$ \\
\hline $\begin{array}{l}\text { Harrell's } \\
\text { C-index } \\
(p)\end{array}$ & 0.60 & $0.60(0.599)$ & $0.62(0.389)$ & $0.62(0.333)$ & $0.62(0.473)$ & $0.62(0.342)$ & $0.62(0.241)$ & $0.62(0.310)$ & $0.62(0.395)$ \\
\hline \multicolumn{10}{|c|}{ High-grade serous } \\
\hline $\begin{array}{l}\text { Adjusted } \\
R^{2}\end{array}$ & 0.099 & 0.100 & 0.1337 & 0.126 & 0.127 & 0.134 & 0.135 & 0.112 & 0.126 \\
\hline BIC & $8,284.92$ & $8,295.007$ & $3,481.144$ & $3,460.403$ & $3,459.172$ & $3,481.147$ & $3,480.754$ & $3,491.962$ & $3,459.309$ \\
\hline $\begin{array}{l}\text { Harrell's } \\
\text { C-index } \\
(p)\end{array}$ & 0.63 & $0.63(0.149)$ & $0.66(0.175)$ & $0.66(0.193)$ & $0.66(0.188)$ & $0.66(0.174)$ & $0.66(0.178)$ & $0.65(0.256)$ & $0.66(0.189)$ \\
\hline \multicolumn{10}{|c|}{ Non-high-grade serous } \\
\hline $\begin{array}{l}\text { Adjusted } \\
R^{2}\end{array}$ & 0.089 & 0.089 & 0.064 & 0.069 & 0.064 & 0.062 & 0.053 & 0.060 & 0.061 \\
\hline $\mathrm{BIC}$ & $3,547.038$ & $16,361.7$ & $1,385.031$ & $1,385.028$ & $1,373.11$ & $1,384.8$ & $1,383.999$ & $1,385.7$ & $1,383.915$ \\
\hline $\begin{array}{l}\text { Harrell's } \\
\text { C-index } \\
(p)\end{array}$ & 0.65 & $0.66(0.643)$ & $0.64(0.213)$ & $0.64(0.193)$ & $0.64(0.164)$ & $0.64(0.186)$ & $0.65(0.094)$ & $0.65(0.124)$ & $0.65(0.208)$ \\
\hline
\end{tabular}

Null model included the predictor's age, education, race, smoking, alcohol consumption, physical activity, parity, family history of ovarian cancer, family history of breast cancer, hormone therapy. Adjusted $R^{2}$ : explained variation

BIC: Bayes Information Criterion. $p$ : $p$ value for difference of Harrell's C vs null model

weight and body fatness changes over the lifecourse are warranted $[39,40]$. Our study also lacked updated information on anthropometric measurements during follow-up.
Another drawback is the low number of cases by ovarian cancer subtype and a lack of statistical power to test for effect modification. 
In summary, results from this prospective study of postmenopausal women do not support associations between measures of central obesity and body fat distribution and risk of ovarian cancer.

Author contributions Conception and design: SEB, IS, and MFL. Development of methodology: SEB, IS, MN, and MFL. Acquisition of data (provided animals, acquired and managed patients, provided facilities, etc.): BT and MFL. Analysis and interpretation of data (e.g., statistical analysis, biostatistics, computational analysis): SEB, IS, and MN. Writing, review, and/or revision of the manuscript: SEB, IS, BT, MN, CM, and MFL. Administrative, technical, or material support (i.e., reporting or organizing data, constructing databases): BT, MN, and MFL. Study supervision: MFL.

Funding Open Access funding enabled and organized by Projekt DEAL.

Data availability Data are available upon request from https://www. aarp.org/forms/research-dataset-request-form/.

Code availability Code is available upon request from the corresponding author.

\section{Compliance with ethical standards}

Conflict of interest No potential conflicts of interest were disclosed.

Open Access This article is licensed under a Creative Commons Attribution 4.0 International License, which permits use, sharing, adaptation, distribution and reproduction in any medium or format, as long as you give appropriate credit to the original author(s) and the source, provide a link to the Creative Commons licence, and indicate if changes were made. The images or other third party material in this article are included in the article's Creative Commons licence, unless indicated otherwise in a credit line to the material. If material is not included in the article's Creative Commons licence and your intended use is not permitted by statutory regulation or exceeds the permitted use, you will need to obtain permission directly from the copyright holder. To view a copy of this licence, visit http://creativecommons.org/licenses/by/4.0/.

\section{References}

1. Wild CP, Weiderpass E, Stewart BW (2020) World cancer report 2020. International Agency for Research on Cancer, Lyon

2. Aune D, Navarro Rosenblatt DA, Chan DS et al (2015) Anthropometric factors and ovarian cancer risk: a systematic review and nonlinear dose-response meta-analysis of prospective studies. Int J Cancer 136:1888-1898

3. Kalliala I, Markozannes G, Gunter MJ et al (2017) Obesity and gynaecological and obstetric conditions: umbrella review of the literature. BMJ 359:j4511

4. Olsen CM, Nagle CM, Whiteman DC et al (2013) Obesity and risk of ovarian cancer subtypes: evidence from the Ovarian Cancer Association Consortium. Endocr Relat Cancer 20:251-262

5. Wentzensen N, Poole EM, Trabert B et al (2016) Ovarian cancer risk factors by histologic subtype: an analysis from the ovarian cancer cohort consortium. J Clin Oncol Off J Am Soc Clin Oncol $34: 2888-2898$
6. World Cancer Research Fund International, American Institute for Cancer Research (2018) Diet, nutrition, physical activity and cancer: a global perspective. Third expert report.

7. Harding JL, Shaw JE, Anstey KJ et al (2015) Comparison of anthropometric measures as predictors of cancer incidence: a pooled collaborative analysis of 11 Australian cohorts. Int J Cancer 137:1699-1708

8. Freisling H, Arnold M, Soerjomataram I et al (2017) Comparison of general obesity and measures of body fat distribution in older adults in relation to cancer risk: meta-analysis of individual participant data of seven prospective cohorts in Europe. Br J Cancer 116:1486-1497

9. Arnold M, Leitzmann M, Freisling $\mathrm{H}$ et al (2016) Obesity and cancer: an update of the global impact. Cancer Epidemiol 41:8-15

10. Lahmann PH, Cust AE, Friedenreich CM et al (2010) Anthropometric measures and epithelial ovarian cancer risk in the European Prospective Investigation into Cancer and Nutrition. Int J Cancer 126:2404-2415

11. Ma X, Beeghly-Fadiel A, Shu XO et al (2013) Anthropometric measures and epithelial ovarian cancer risk among Chinese women: results from the Shanghai Women's Health Study. Br J Cancer 109:751-755

12. Minlikeeva AN, Moysich KB, Mayor PC et al (2018) Anthropometric characteristics and ovarian cancer risk and survival. Cancer Causes Control CCC 29:201-212

13. Schatzkin A, Subar AF, Thompson FE et al (2001) Design and serendipity in establishing a large cohort with wide dietary intake distributions: the National Institutes of Health-American Association of Retired Persons Diet and Health Study. Am J Epidemiol 154:1119-1125

14. Bergman RN, Stefanovski D, Buchanan TA et al (2011) A better index of body adiposity. Obesity 19:1083-1089

15. Krakauer NY, Krakauer JC (2012) A new body shape index predicts mortality hazard independently of body mass index. PLoS One 7:e39504

16. Wu F, Ho V, Fraser BJ et al (2005) (2018) Predictive utility of childhood anthropometric measures on adult glucose homeostasis measures: a 20-year cohort study. Int J Obes 42:1762-1770

17. Michaud D, Midthune D, Hermansen S et al (2005) Comparison of cancer registry case ascertainment with SEER estimates and self-reporting in a subset of the NIH-AARP Diet and Health Study. J Regist Manag 32:70-75

18. Fritz A, Percy C, Jack A et al (2000) International classification of diseases for oncology: ICD-0, 3rd edn. World Health Organization, Geneva

19. Carcangiu M, Kurman RJ, Carcangiu ML, Herrington CS (2014) WHO classification of tumours of female reproductive organs. International Agency for Research on Cancer, Lyon

20. Dixon SC, Nagle CM, Thrift AP et al (2016) Adult body mass index and risk of ovarian cancer by subtype: a Mendelian randomization study. Int J Epidemiol 45:884-895

21. Kossaï M, Leary A, Scoazec J-Y, Genestie C (2018) Ovarian cancer: a heterogeneous disease. Pathobiology 85:41-49

22. Matulonis UA, Sood AK, Fallowfield L, Howitt BE, Sehouli J, Karlan BY (2016) Ovarian cancer. Nat Rev Dis Primers 2:16061

23. Reid BM, Permuth JB, Sellers TA (2017) Epidemiology of ovarian cancer: a review. Cancer Biol Med 14:9-32

24. VanderWeele TJ (2019) Principles of confounder selection. Eur J Epidemiol 34:211-219

25. Harrell F (2015) Regression modeling strategies: with applications to linear models, logistic and ordinal regression, and survival analysis. Springer, Cham

26. O'Quigley J, Xu R, Stare J (2005) Explained randomness in proportional hazards models. Stat Med 24:479-489

27. Royston P, Parmar MK (2002) Flexible parametric proportionalhazards and proportional-odds models for censored survival 
data, with application to prognostic modelling and estimation of treatment effects. Stat Med 21:2175-2197

28. Antolini L, Nam B-H, D'Agostino RB (2004) Inference on correlated discrimination measures in survival analysis: a nonparametric approach. Commun Stat Theory Methods 33:2117-2135

29. Keogh RH, White IR (2014) A toolkit for measurement error correction, with a focus on nutritional epidemiology. Stat Med 33:2137-2155

30. Maukonen M, Männistö S, Tolonen H (2018) A comparison of measured versus self-reported anthropometrics for assessing obesity in adults: a literature review. Scand J Public Health 46:565-579

31. McAdams MA, Van Dam RM, Hu FB (2007) Comparison of selfreported and measured BMI as correlates of disease markers in US adults. Obesity (Silver Spring, Md.) 15:188-196

32. Rimm EB, Stampfer MJ, Colditz GA, Chute CG, Litin LB, Willett WC (1990) Validity of self-reported waist and hip circumferences in men and women. Epidemiology (Cambridge, Mass.) 1:466-73

33. McAdams MA, Van Dam RM, Hu FB (2007) Comparison of selfreported and measured BMI as correlates of disease markers in US adults. Obesity 15:188

34. Danaei G, Robins JM, Young JG, Hu FB, Manson JE, Hernan MA (2016) Weight loss and coronary heart disease: sensitivity analysis for unmeasured confounding by undiagnosed disease. Epidemiology (Cambridge, Mass.) 27:302-310
35. Flegal KM, Graubard BI, Yi SW (2017) Comparative effects of the restriction method in two large observational studies of body mass index and mortality among adults. Eur J Clin Investig 47:415-421

36. Jochem C, Schlecht I, Leitzmann M (2018) Epidemiologic relationship between obesity and ovarian cancer. In: Berger NA, Klopp AH, Lu KH (eds) Focus on gynecologic malignancies. Springer, Cham, pp 21-30

37. Kyrgiou M, Kalliala I, Markozannes G et al (2017) Adiposity and cancer at major anatomical sites: umbrella review of the literature. BMJ 356:j477

38. Qian F, Rookus MA, Leslie G et al (2019) Mendelian randomisation study of height and body mass index as modifiers of ovarian cancer risk in 22,588 BRCA1 and BRCA2 mutation carriers. $\mathrm{Br}$ J Cancer 121:180-192

39. Huang T, Tworoger SS, Willett WC, Stampfer MJ, Rosner BA (2019) Associations of early life and adulthood adiposity with risk of epithelial ovarian cancer. Ann Oncol 30:303-309

40. Keum N, Greenwood DC, Lee DH et al (2015) Adult weight gain and adiposity-related cancers: a dose-response meta-analysis of prospective observational studies. J Natl Cancer Inst 107:djv088

Publisher's Note Springer Nature remains neutral with regard to jurisdictional claims in published maps and institutional affiliations. 